\title{
Six Questions on Topology in Theoretical Chemistry
}

Paul L. Ayers ${ }^{\mathrm{a}}$, Russell J. Boyd ${ }^{\mathrm{b}}$, Patrick Bultinck ${ }^{\mathrm{c}}$, Michel Caffarel ${ }^{\mathrm{d}}$, Ramon Carbó-Dorca , Mauro Causáf $^{\mathrm{f}}$, Jerzy Cioslowski $^{\mathrm{g}}$, Julia Contreras-Garcia ${ }^{\mathrm{h}}$, David L. Cooper ${ }^{\mathrm{i}}$, Philip Coppens ${ }^{\mathrm{j}}$, Carlo Gatti ${ }^{\mathrm{k}}$, Simon Grabowsky ${ }^{\mathrm{l}}$, Paolo Lazzeretti $^{\mathrm{m}}$, Piero Macchi ${ }^{\mathrm{n}}$, Ángel Martín Pendás ${ }^{\mathrm{o}}$, Paul L. A. Popelier ${ }^{\mathrm{p}, \mathrm{q}}$, Klaus Ruedenberg ${ }^{\mathrm{r}}$, Henry Rzepa ${ }^{\mathrm{s}}$, Andreas Savin ${ }^{\mathrm{h}}$, Alexander Sax ${ }^{\mathrm{t}}$, W. H. Eugen Schwarz ${ }^{\mathrm{u}}$, Shant Shahbazian ${ }^{\mathrm{v}}$, Bernard Silvi ${ }^{\mathrm{h}, *}$, Miquel Solàw, Vladimir Tsirelson ${ }^{\mathrm{x}}$

\footnotetext{
${ }^{a}$ Department of Chemistry and Chemical Biology, McMaster University, 1280 Main Street West, L8S 4M1, Hamilton, Ontario, Canada

${ }^{b}$ Department of Chemistry, Dalhousie University, Halifax, Nova Scotia, Canada B3H $4 R 2$

${ }^{c}$ Department of Inorganic and Physical Chemistry, Ghent University, Krijgslaan 281 (S3), B9000 Ghent, Belgium

${ }^{d}$ Laboratoire de Chimie et Physique Quantiques, CNRS-IRSAMC Université de Toulouse, France

${ }^{e}$ Institute of Computational Chemistry, University of Girona, Campus de Montilivi, 17071 Girona, Spain

${ }^{f}$ Dipartimento di Chimica Paolo Corradini, Universitá degli Studi di Napoli “Federico II", Via Cintia, 80126 Napoli, Italy

${ }^{g}$ Institute of Physics, University of Szczecin, Wielkopolska 15, 70-451 Szczecin, Poland

${ }^{h}$ Sorbonne Universités, UPMC, Univ Paris 06, UMR 7616, Laboratoire de Chimie Théorique, case courrier 137, 4 place Jussieu, F-75005 Paris, France

${ }^{i}$ Department of Chemistry, University of Liverpool, Liverpool L69 7ZD, United Kingdom

${ }^{j}$ Chemistry Department, University at Buffalo, SUNY, Buffalo, New York 14260-3000, United States

${ }^{k}$ CNR-ISTM Istituto di Scienze e Tecnologie Molecolari, via Golgi 19, 20133 Milano, Italy

${ }^{l}$ School of Chemistry and Biochemistry, Chemistry M310, The University of Western Australia, 35 Stirling Highway, Crawley WA 6009, Australia ${ }^{m}$ Dipartimento di Scienze Chimiche e Geologiche, Universitá degli Studi di Modena, via G. Campi 183, 41100 Modena, Italy

${ }^{n}$ Department of Chemistry and Biochemistry, University of Berne, Freiestrasse 3, CH-3012 Berne, Switzerland

${ }^{o}$ Departamento de Química Física y Analítica, Universidad de Oviedo, E-33006 Oviedo, Spain

${ }^{p}$ Manchester Institute of Biotechnology (MIB), 131 Princess Street, Manchester, M1 7DN, Great Britain

${ }^{q}$ School of Chemistry, University of Manchester, Oxford Road, Manchester, M13 9PL, Great Britain

${ }^{r}$ Department of Chemistry and Ames Laboratory USDOE, Iowa State University, Ames, Iowa 50011,USA

${ }^{s}$ Department of Chemistry, Imperial College London, South Kensington Campus, Exhibition Road, London SW7 2AY, U.K

${ }^{t}$ Department of Chemistry, University of Graz, Heinrichstrasse 28, 8010 Graz, Austria

${ }^{u}$ Theoretical Chemistry Groups at Tsinghua University, Beijing, 100084, China, and Physical and Theoretical Chemistry Laboratory, Faculty of Science and Engineering, University of Siegen, Siegen, 57068, Germany

${ }^{v}$ Faculty of Chemistry, Department of Chemistry, Shahid Beheshti University, G.C., Evin, Tehran, Iran

${ }^{w}$ Institut de Química Computacional i Catàlisi (IQCC) and Departament de Química, Universitat de Girona, Campus de Montilivi, E-17071 Girona, Catalonia, Spain

${ }^{x}$ Department of Quantum Chemistry, Mendeleev University of Chemical Technology, Miusskaya Square 9, 125047 Moscow, Russia
}

\section{Abstract}

The paper collects the answers of the authors to the following questions:

- What is the significance of the topological approach?

- Can new chemical concepts be found by a topological approach?

- What is the status of a chemical concept within a topological approach?

- Should topological approaches provide measurable quantities?

- Is it possible to predict the outcome of a topological approach without performing a calculation on a computer?

- What are new domains for which topological approaches would be useful?

\footnotetext{
${ }^{*}$ Corresponding author

Email address: silvi@lct.jussieu.fr (Bernard Silvi)
} 
Andreas Savin and Bernard Silvi. We have been asked by the editors to write an introductory article to this special issue of Theoretical and Computational Chemistry. They kindly left us the choice between a short review and anything else. We retained the second option and decided to produce a collective paper in which a panel of scientists were invited to give their opinions on the topological approaches in Theoretical Chemistry. In march 2014, a letter giving the rules of the game was sent to about sixty quantum chemists involved in the development and applications of topological methods. We asked the contributors to answer as concisely as possible six questions. A wiki page was created on the web site of the Laboratoire de Chimie Théorique enabling each contributor to leave his answers and update them in accordance with the answers of the others. The wiki page was closed on May 31. We did not edit the answers, as they reflect the diversity of opinion that characterizes scientific discussions. During the editing process we received the answers of Piero Macchi which are welcome in this article.

Henry Rzepa. George Whitesides is one of those who have urged the community to make their science more accessible (his solution was video). I too find experimenting with different formats refreshing, and part of the process of making our science more accessible. I myself took part in a "trialogue" with two others where again a serial format illustrating our thoughts over a six month period was captured[1]. I recently attended a celebration of the work of the pioneering Jean-Claude Bradley (who died at an absurdly young age) where he introduced the concept of "open notebook science" in getting the community to collaborate on new drugs, and to curate a melting point database. There are many other experiments of this type out there; some (many?) will not persist, but I think as a community it is our obligation to do what Whitesides urges and communicate better. I regard the current "interesting object" as yet another of these experiments. In a sense, since it started on a Wiki, that this too was an experiment in notebook science (albeit not quite open in the manner JC envisaged). We have done something very similar for $>10$ years now with our undergraduate students, and they seem to relish it.

Shant Shahbazian. This contribution is freezing a snapshot of the history of theoretical chemistry. It reveals how people "really" think about this field now. Why not leaving a text for historians of quantum chemistry in next generation? I am wondering what will be our answers twenty years in future.... Let not be too sensitive to rigor in this contribution. I guess others will also enjoy reading this strange text. It was a bold (and now I think really fruitful) decision made by the guest editors and may open the door for such contribution in future.

\section{What is the significance of topological approach?}

Is it the development of functions of spatial (or momentum) coordinates and the further discussion of their representation? or is it the use of mathematical methods belonging to topology (like singularity theory) in order, for instance, to carry out the analysis of a function?

Paul Ayers. I think that either of the two choices is plausible, but I usually think of the first topic: finding new quantities that are amenable to "chemical topology" are (inappropriately? appropriately? I'm not sure) stagnant: once one has a new function, the next steps are often clear. Obviously a mathematician (or mathematically-inclined chemist) who was well-versed in topology might find new and interesting approaches, but (to me) the simplest approaches seem to work well.

W. H. Eugen Schwarz. The question has two aspects, i) a semantic one and ii) that of purpose and meaning.

i) Physicists often insist that words have and should have only the meaning that is used by themselves. A nice example is "interference". In physics it means that the superposition of waves results in a new interference pattern; in analytical and pharmacological chemistry it means the change of an effect due to the presence of some other substance; in chromosomal genetics, in cognition and teaching psychology, in chess, etc. "interference" has other meanings. I hold that chemists should stay with their use of the word "topological" as a somewhat vague concept (rather common in chemistry) indicating the connection of physical or imagined points or regions of microscopic matter, either in the classical sense or represented by some quantal matter field function. Since in Greek tóros (topos) means place,

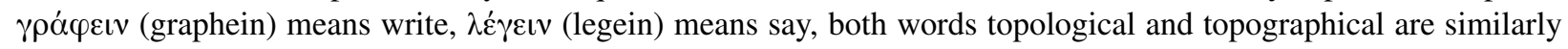
appropriate for the field under discussion.

ii) Consequently, the topological approach is the analysis of points or functions in some space, these points or functions characterizing the chemical substances. Various tools may be used, including also the concepts and methods 
of mathematical topology. As spaces, one may investigate the position space, the momentum or linear and angular momentum space, the classical phase space or one of its quantum analogs, chemical similarity spaces and so on. The aim is to extract chemically useful compact indices and more extended information. The aim should not be replacing chemistry by sterile numerology. The aim should be to find additional, or more compact or more sharply defined descriptors for chemical substances. Since chemistry has two aspects: Many (stationary) Substances, and Changing the substances, the topological approach should be applied to the investigation of substances and of reactions. Note that in the Chinese language, the word for Chemistry ( 化学, huà-xué) literally means "Science of Change". So far most of the topological approach has concentrated on the description of stationary substances, i.e. molecules and crystals in equilibrium, and be it under pressure. The significance of the topological approach in chemistry could be greatly extended in the future, if one succeeds in efficiently handling chemical processes. Thereby, two goals could be reached. First the description of substances and how they are formed in reactions; and second, it might become possible to explain, why this or that isomer is stable, why this or that one is formed. So far the topological approach has mainly been restricted to describe "how nature is", but not yet extended to explain "why nature is as it is". Summarizing, the topological approach in chemistry is a whole set of approaches including tools from mathematical topology.

Mauro Causá. As a first answer I prefer the second. I think the term "topology" is not appropriate for defining the present field, so the use of physical observables (or quasi observable) for defining unambiguously the chemical phenomenological concepts, even if we make geometrical analysis of physical space functions like $\rho(\mathbf{r}), \operatorname{ELF}(\mathbf{r})$ and $P(n, \Omega)$ for finding chemistry in physics. I prefer to reserve the term topology to the use of mathematical topology analysis to network of "bonds", a field that seems newly interesting, if applied not only to poly aromatics.

Paolo Lazzeretti. As regards the first question I would emphasize that topology is a branch of mathematics, born as "analysis situ", and applied nowadays to physics, chemistry and biology. In chemistry, vague or ill-defined concepts, say "aromaticity", can be dealt with allowing for topological indices which provide quantitative information.

Bernard Silvi. Rather than a topological approach there are topological approaches to definite topics in which a mathematical method belonging or related to topology is explicitly or intuitively used to build a model or to analyse a set of data. As non exhaustive examples: graph theory has been applied to molecular structure, dynamical system theory (enabling to extract qualitative pieces of information from numbers) to the study of different scalar fields (BO energy surfaces, electron density, MESP, ELF), knot theory to the study of chirality, fractal theory to colloïds and dendrimers, chaos theory to oscillating reactions. Interesting reviews have been written by Babaev[2] and Cramers[3].

Russell Boyd. It is a shame that Richard Bader is no longer a participant in the discussion. He could be relied upon to enter all debates and discussions with his unique style and intense passion for science. Life was never dull in Richard Bader's presence. The first answer probably comes closer to capturing the view of chemists, while the second is closer to the purview of mathematicians. I sometimes think chemists are thinking more in terms of the topography of the electron density than topological analysis of the electron density and other quantities.

Patrick Bultinck. If we consider the first choice a more chemistry oriented one and the second a more mathematical one, I still think both are needed. It would be good to have the unbiased view of a mathematician (or anybody that lacks the "chemical bias") on topological analysis. If I may consider Richard Bader's AIM theory a topology, I think it is too often forgotten that this is not a pure topological approach. Essentially, these AIM are based on domains where the Schrodinger kinetic energy and the "gradient scalar product" kinetic energies[4] are the same despite the underlying property densities being significantly different (see for example Anderson et al. for some more allowable kinetic energy densities[5]). That they share the same expectation value over an AIM domain is thanks to the disappearance of the Laplacian of the density over this domain[6]. Now, there is an infinite choice of domains that fulfils this, as is immediately clear[7]. However, Bader himself in 1973[8] excluded any gradient line that ends (starts) on a nucleus. Moreover, it is an integral that needs to be zero and not an integral of zero values. So from the very start, a topological analysis was "guided" in an admittedly brilliant way to lead to chemistry. But what if we do not guide it. Who knows what could be found? Second, in all these approaches we attempt to recover chemistry and in this context, there is nothing to be ashamed of to define new functions and then to analyse these. But here also lies a risk. Is a function wrong if it does not reveal what we "want to see"? 
Shant Shahbazian. Topological approach in itself does "not" yield novel mathematical information but simply makes it possible to "magnify" and "concentrate" on some special characteristics of a scalar function namely its extrema using its gradient vector field. For theories like the QTAIM that are interested in such extrema to grasp their basic objects, e.g. topological atom as the basin of attraction of the gradient field of the one-electron density, topological approach is a proper tool to reveal the "relevant" points/surfaces, i.e. bond critical points/inter-atomic surfaces. It is in principle applicable and useful for any theory that is somehow interested in extrema of functions, e.g. the ELF[9]. The Catastrophe theory and other more sophisticate topological approaches, though interesting, have just a secondary/auxiliary role.

Klaus Ruedenberg. The aim in our field is to relate molecular properties to electronic structure, which means electronic densities (1st and 2nd order) and energy contributions from various parts of a molecule. Topological reasoning is one of the tools that can sometimes be helpful in identifying "macro"-features without being distracted by "micro"details. More sophisticated topological methods are only interesting if they enhance the capabilities of extracting basic physical implications that are embedded in i specific electronic wave functions.

Carlo Gatti. In my view, topological approach has a broader meaning than that covered by either of the two views that have been proposed above. Moreover, it is not just represented by their sum. It is rather connected to the

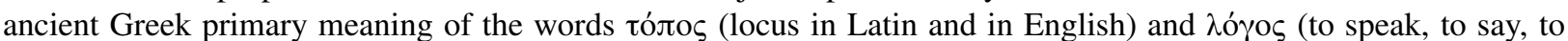
tell about), thereby indicating an approach that puts a special emphasis on the local properties of functions of spatial (or momentum) coordinates, to reach some, possibly useful, insight on the properties of a system. Functions can be selected, for instance, as for their ability to convey chemical information tied to some simplistic chemical model. The loci where to study these functions may be suggested by singularity theory. Local properties may be then related either to global, "integral" properties of (a part of ) the system, or also to the features of convenient model "objects" describing the system. An example of integral property is the energy or the number of electrons of a portion of system, one of a convenient model object is the "chemical bond" or its representation in terms of simple diagrams (e.g. Lewis structures).

Julia Contreras-Garcia. As I see it, what we are referring to is chemical topology. So the application of mathematical topology concepts to recover, and hopefully expand, classical chemical concepts. Thus, to our community 2) does not exist without 1), because we need a function of some kind to apply the mathematical concepts to. Topology is a well adapted tool thereto because it enables us to go from point-wise analysis, which scapes our understanding and which we do not know how to relate to macrocopic properties of the system to something we can understand, dissect, and build -predictive- concepts upon (I tried to avoid mixing questions, but I could not resist).

Simon Grabowsky. I like the idea of Russel Boyd to distinguish between topography and topology. Every scalar function based on spatial or momentum coordinates has a topography. Maybe we could say that topological analysis in the sense we mean it in chemistry is the use of mathematical tools and procedures in order to break down the three-dimensional topography of a physically meaningful function into interpretable properties in a quantitative way. Hence, I think the topological approach is not the development of new functions, but the described way of analysing new or old functions - any scalar function - to make them accessible to chemical interpretation.

Jerzy Cioslowski. Strictly speaking, the term "topological" should be reserved for: 1) analysis of functions in the Cartesian or momentum space (such as the electron density or ELF) that is based upon identification of critical points and the relations ("connectivities") between them and 2) construction of Hamiltonians that use only atomic connectivities as input (as in the Hückel theory a.k.a. chemical graph theory). For example, QTAIM really involves two steps: 1) topological analysis of electronic density that cannot be disputed from mathematical standpoint and 2) identification of the topological features of electron density with chemical concepts that is subject to rather arbitrary interpretation (recall the raging discussion on weak bonds/“negative bonds"/repulsive bonds in e.g. planar biphenyl).

Paul Popelier. To me the significance of the "topological approach" has always been its ability to partition a system into well-defined regions. This is crucial because many questions in chemistry boil down to a partitioning issue. The way the "topological approach" partitions is minimal and I like that; moreover, it has a visual appeal that has not be fully exploited (see movies in drug design). The partitioning is minimal because it is parameter-free and 
does not assume anything, such as a reference system, or a priori sphericity. The gradient vector is all one needs to go ahead with partitioning. "Minimal" should not be confused with "simple" (see my little story on the ellipse versus the circle in solar system mechanics in the preface of reference[10]). I like minimal approaches because they generate a lot of information without imposing anything. Putting the idea of gradient vector field upfront is what binds the various topological approaches (QTAIM (both the electron density and its Laplacian), ELF, IQA, ..., See Box 8.1 in reference[11]), which is one of the reasons for the overarching name "Quantum Chemical Topology" (QCT)[12, 13], which really stands for "the topological approach". If forced to choose between the two subquestions above, I therefore veer towards the second one. The separatrices that QCT discovers are part of a wider language, that of dynamical systems, which includes critical points etc. More advanced and related mathematical concepts (e.g. Riemannian curvature) should be explored in connection with QCT.

Ángel Martín Pendás. A point of view which is floating around in many of the answers provided distinguishes between the functions that are supposed to convey chemically relevant information (either in real or momentum space) and the mathematical tools (borrowed from topology, graph theory, catastrophe theory, etc) used to data mine them. To me, the "topological approach" provides a unique way to discover specific points in (real or momentum) space -the local stance, with its critical points correlating with all sort of molecular properties-, and at the same time to coarse-grain or partition the space into regions. This leads to the global stance, with integrated properties that add to the total quantum-mechanical expectation values. In a sense, it is this bimodality that makes the approach so useful, since one may switch from one stance to the other at convenience without leaving the general framework.

Vladimir Tsirelson. I prefer the first answer. I also think that neither "topological approach" nor "Atoms in molecules" and "Quantum chemical topology" are not proper names of the field. I would suggest the following: "Quantum topological theory of molecular and crystal structure".

Michel Caffarel. To me the key issue is to construct simple mathematical representations allowing chemists/physicists to visualize and rationalize what happen in a complex molecular system described by a highly structured and entangled $3 \mathrm{~N}$-dimensional wavefunction through the use of much more simpler 3D functions and topological analysis. Due to this loss of information, the notion of chemical bond in ordinary space, and more generally most chemical concepts, appear to me as necessarily and intrinsically not well defined, although useful as any taxonomy. Localization functions and topology can help (eventually a lot) but within these strict limits. Hopefully, the problem can be reduced to lower dimensions through two- and one-body densities. Localization functions in close relation to the two-body matrix are thus attractive. An example is the electron pair localization function (EPLF) introduced in [14, 15] that is directly related to the two-body density matrix and that is usable for any type of wavefunctions and excited states. Another fundamental aspect related to intricacy and delocalization of the highly multi-dimensional wavefunction is that any partition in 3D space into well-defined regions seems hardly compatible with quantum mechanics. Some fuzzy aspects in domains taking their source in multi-particle correlations should appear. In that respect I find the idea of Savin of defining overlapping domains of maximal probability for a given number of electrons very interesting (see [16], and [17] also).

Alexander Sax. The topological approach helps chemists to feel like mathematicians: Just call everything related to geometry (distances, angles) or graph theory (arcs connecting nodes) topology. And you need not care about horrifying aspects like filters, compactness, homotopy etc.

Piero Macchi. In Chemistry, a topological approach is a link between a simple concept (structure, reaction mechanism, functionality etc.) and an observable. It can be used to understand chemical bonding, to anticipate chemical reactions or to rationalize materials properties. Mathematically, a topological approach involves both the analysis of a function and its representation in terms of simple elements.

\section{Can new chemical concepts be found by a topological approach? Can traditional chemical concept definitions} be improved by topological approaches?

Henry Rzepa. I will answer this perhaps in an unexpected manner, where I am redefining the topological approach to chemistry rather more broadly. Our interest in topological techniques such as QTAIM and ELF arose because we 
wanted to study the properties of aromatic systems. Conventional approaches for assessing aromaticity do not work as easily if the aromatics are topologically non planar. We discovered the wonderful topological world of knots and links, and the theorems associated with them (see figure 1). The White/Călugăreanu/Fuller theorem relates to the geometry

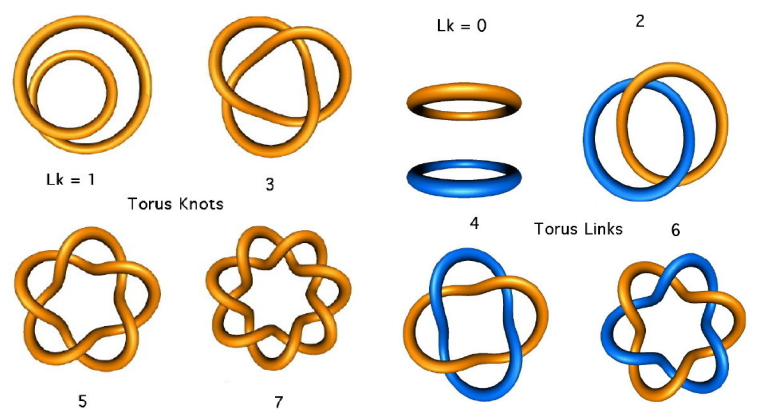

Figure 1: Torus knots and links of relevance to twisted conjugated cyclic molecules.

of closed twisted ribbons by a topological invariant known as the Linking number, where $L k=T w+W r$, where $W r$ or Writhe measures the extent to which coiling of the central curve has relieved local twisting $T$ of the ribbon and $T w$ is $\int T$. The $\pi$-density $\rho(\mathbf{r}) \pi$-manifold of benzene for example can be described with a linking number of 0 , but the interesting linking numbers are $\neq 0$. For example, a Möbius $\rho(\mathbf{r}) \pi$-manifold has a linking number of $\pm 1 \pi$, and a three dimensional lemniscate is \pm 2 . In chemistry, these topological invariants are also chiral descriptors, thus -1 is the mirror image/enantiomer of +1 . In that sense, the linking number is a manifestation of the Cahn-Ingold-Prelog $\mathrm{M} / \mathrm{P}$ helical notation for higher-order topologies.

Molecules exhibiting these properties are in fact well known; many extended porphyrins adopt such topologies (see C. S. M. Allan and H. S. Rzepa[18] or H. S. Rzepa[19]. It turns out that this allows a general extension of the simple Hückel rules for aromaticity: For even values of the linking number (including zero), the $\pi$-density $\rho(\mathbf{r})$ manifold will exhibit aromaticity/diatropicity if populated with $4 n+2 \pi$-electrons and antiaromaticity/paratropicity for values of $4 n$ under thermal conditions. For odd values of the linking number, the $\pi$-density $\rho(\mathbf{r})$ manifold will exhibit aromaticity/diatropicity for values of $4 n$ and antiaromaticity/paratropicity if populated with $4 n+2 \pi$-electrons under thermal conditions. So to answer the question directly "Can traditional chemical concept definitions be improved by topological approaches?", yes one can use these topological approaches to gain insight into the aromaticity of twisted (non-planar) conjugated molecules. And one can achieve a fusion of two classical 19th century concepts of aromatic molecules and of chirality since as I noted above, manifolds with non-zero linking numbers are chiral. I close by saying that the above also provides an answer to questions 5 and 6. More details can be found at S. M. Rappaport and H S. Rzepa[20]

Paul Ayers. Yes, and Yes. The latter question is most apparent and in my narrow field (conceptual DFT) one often takes "traditional" (or at least quasi-traditional) concepts and applies the topological toolbox to them. As for new chemical concepts, I hope so, and I have faith that it is true. For example, in our work on using machine-learning to identify molecular descriptors, it seems likely that a "topological signature" that is common to some phenomenon could be identified. (This is to say, it is possible that the descriptor(s) one finds when trying to organize a broad range of chemical phenomena into classes will be topological.) That said, I don't know how many compelling new concepts have been identified; it seems to me that the most powerful and useful chemical concepts are half a century old, or older.

Mauro Causá. Yes, The so called topological approaches (QTAIM, ELF and MPD) can be definite answers to the problem of understanding the chemical phenomenological concepts within a physical framework.

Vladimir Tsirelson. Yes. It is really doing very often. 
Paolo Lazzeretti. That's precisely what I expect from my "topological" approach to "chemical ideas". For instance, the interpretation of aromaticity on the magnetic criterion is based on a topological object named "stagnation graph". The singular branching points of this graph are analyzed in connection with the notion of "delocalized electron cloud".

Russell Boyd. I would answer yes to both questions, but I am not entirely happy with the current state of affairs. Let me explain with the example of electronegativity, which is introduced to students at an early stage in their study of chemistry. Electronegativity is a chemical concept that is used to rationalize many observations and is a major part of the vocabulary of chemistry, but the theoretical basis for the concept is far from being satisfactory. In 1988, Ken Edgecombe and I tried to evaluate atomic and group electronegativities from the topological properties of the electron density distributions of molecules[21, 22]. Although our manuscript was published in a prominent journal, I have always had the feeling that it should be possible to produce a better approach. Electronegativity is only one of many chemical concepts for which I think there is a need for a more rigorous theoretical foundation. If that foundation is possible, then I think it is mostly likely to come from the application of topological approaches to the results of quantum mechanics.

Patrick Bultinck. Do we really need even more concepts? I'd be happier if we concentrated first on getting these right or at least linked to something quantum chemically/mechanically better defined. Topology may help in this but I think we need to realize that many of these concepts were never meant to be quantified. Take aromaticity, there is discussion on what is more aromatic; e.g. benzene or the pentadienyl anion, but we quite often forget to define closely the meaning of aromatic. To me it is the property of having a delocalized system (also to be properly defined first) and the property of exhibiting a current density of right nature (read: like in benzene) but to many others it is the property of having a such and such NICS value. So, new concepts might make everything even more diffuse. Narrowing down, or describing more accurately existing concepts is a good option but remember Mulliken's statement that the more we know and compute, the more concepts disappear[23]: are we prepared, willing, capable of accepting that ? I sure do hope so but it'll be a hard job to ask "regular chemists" to throw away a tool that is not broken in their view. Even R.G. Parr has struggled with this question, given his statement "Accurate calculation is not synonymous with useful interpretation. To calculate a molecule is not to understand it" that supports the conceptual approach[24]. Maybe now, with even better methods and higher accuracy than in 1965, we could ask this question again instead of coming up with more concepts.

Shant Shahbazian. The general answer to the first question is "no". Topological approach is indeed a mathematical tool to "extract" chemical information from wavefunctions in real space making a "bridge" between quantum mechanics (QM) and chemistry. But, to make a bridge, one needs to the both sides. Chemical concepts are introduced in chemical discourse based on their significance in chemistry not emerging from QM. We know there are atoms in molecules from chemical experiences (not QM!) then seeking to extract them from wavefunctions "inventing" the QTAIM and its topological apparatus. This is also true in the case of ELF methodology that assumes there are lone pairs, then seeking them using topological apparatus. The answer to the second question is just a conditional "yes". Yes, if topological procedure is part of a comprehensive theory that "redefines" the relevant chemical concept in a rigorous manner like "the" QTAIM. It must be stressed that topological approach is "not" in itself a theory but always part of a theory/methodology that aims to make the mentioned bridge. Thus, its success depends on the main theory[25].

Julia Contreras-Garcia. I would say that, like any other theoretical development, topological approaches should be able to 1) recover previous (classical chemical) concepts and 2) expand them (otherwise, we would not need them!). The recovery should ideally be done at the qualitative and quantitative level, not only we have to find bonds, but we should be able to find a charge for it which fits into classical theories. In this matching, we should concentrate in text-book examples. Once this is done to a reasonable (which of course is quite ambiguous) level, the 'extension' phase should come in. E.g. It is very good to see lone pairs where we know lone pairs are, but where are the lone pairs in the electrides, for example. This is a very neat example that I always use with students: sodium under very high pressure loses its metallic properties, i.e. it is an insulator. This escapes classical approaches, however, ELF is able to show very clearly that electrons localize in the structural voids as pseudo anions, thus forming an insulating pseudo-ionic solid, whereas it shows a very clear metallic structure at ambient pressure (and is thus conducting at ambient conditions). 
Simon Grabowsky. The discussion seems to develop into the direction (especially Patrick Bultinck and Shant Shahbazian) that topological analysis should be unrelated to the discovery, confirmation, improvement or refutation of chemical concepts because chemical concepts are meant to be broad with fuzzy boundaries. This allows their universal applicability and enhances chemical understanding beyond the level of numerical details. Within this train of thoughts, topological analysis should rather provide a framework to compare properties of different compounds and enable correlation to their chemical function, i.e. bridging quantum mechanics/experiments with chemical concepts. As an example, I must think of the use of pure QTAIM[26] as well as the related Source Function[27] to define different classes of hydrogen bonds, i.e. to "improve" the fuzzy concept of hydrogen bonds by introducing sharp boundaries between hydrogen bond types. These ideas of classification are certainly very interesting, and maybe they are useful for correlations, but do they enhance our understanding of the underlying chemical concept?

Carlo Gatti. Yes, it occasionally happens that the topological approach discovers new chemical concepts or that it simply finds a sharper definition/classification of loosely categorized physico/chemical features. One odd, but interesting case, is that of the occurrence of non-nuclear attractors (NNAs) of the electron density gradient field that I, as a young "reckless" fellow, introduced in QTAIM, along with Pacchioni and Fantucci, while working on the chemical bond features in Li clusters[28]. QTAIM had not to be modified, but simply generalized to systems where, besides the usual atomic basins enclosing a nucleus, "strange" basins bounded by the usual zero-flux surfaces but not enclosing a nucleus also occur. When I told Richard (Bader) about NNAs, he stared at me clearly disconcerted for a while, but after ten minutes or so, he enthusiastically embraced the idea that under some circumstances the electron densities of atoms may rearrange upon combination as to form maxima at positions other than the nuclei and I cooperated with him and his group in publishing a second paper on NNAs[29]. Occurrence of non-nuclear maxima in the density has then been elegantly rationalized by A. Martín Pendás et al.[30]. They have shown that each homonuclear diatomics has a stability window of internuclear distances for a midpoint non-nuclear maximum to occur. Therefore, rather than being an oddity, non nuclear maxima are a normal step in the chemical bonding of homonuclear groups, if analysed in the appropriate range of internuclear distances. For most elements, this range occurs however far away from the stable geometry under normal thermodynamic conditions, but such range may easily reached under (high) pressure, where the occurrence of NNAs is very frequent (for instance in the insulating structural phase of $\mathrm{Na}$ [31]. The NNA concept has now been largely recognised and put in correspondence with F centers in crystals[32, 33], or as a mark of solvated electrons (e.g. [34]), or to the necessity of concentrating electrons in the interstitial regions of (elemental) solids (e.g. [31]) to contrast the application of a large external pressure and to so lower the total energy. Such electron density displacement may be also envisaged as a promotion, under pressure, of atomic states of higher angular momentum than the ground state.

Klaus Ruedenberg. Topology is an analysis of relative positions of objects with respect to each other in space. It contains no information whatsoever regarding the physics that drives objects to move or be where they are in space. Manifestly, topology per se cannot yield physical concepts. On the other hand, the complexity of molecular and, in particular, super-molecular structures frequently embodies certain spatial motives that re-occur under a variety of conditions. Some of these motives can be characterized by topological identifiers, which some may call chemical concepts. Recall in this context that Dirac denounced the "pest of group theory" because it contains no physical concepts. Yet the theoretical concepts of point groups have proven very useful, even though they contain no physical concepts. Recall also that the basic physical concept of constants of motion is a consequence of the mathematical invariance theorem of Emmy Noether. I doubt whether topology can add to elucidating basic bonding concepts. It has not done so far. But it may be well positioned to organize the understanding of bonding patterns in complex structures, e.g. nano-structures.

Paul Popelier. Yes to both questions but we need to be brave and follow the full consequences of this approach through to the end. With enough work done, the whole of chemistry could be recast into a nice and coherent framework based on the topological approach. Space allows me to mention only two examples. In work to be published we show that the rotation barrier in biphenyl (from planar to equilibrium at the central torsion angle of $\sim 45^{\circ}$ ) is not due to the textbook explanation of steric hindrance between the bay hydrogens $\mathrm{H} 2 \cdots \mathrm{H} 2^{\prime}$. Instead, a full topological partitioning of the molecular energy (using the topology of the electron density) shows that this barrier is due to the destabilised intra-atomic energies in the planar conformation of only four atoms: $\mathrm{C} 2, \mathrm{H} 2, \mathrm{C}^{\prime}$ and $\mathrm{H} 2{ }^{\prime}$. In fact, the interaction 
between the two "clashing hydrogens" is actually stabilising, resulting from substantial exchange energy between them. As a second example, imagine that you have never seen a hydrogen bond. You would soon see one via its topological signature in a plot of interatomic exchange energy versus internuclear distance. This is clear from Figure 2 in reference[35] where both the $\mathrm{O} \cdots \mathrm{O}$ and $\mathrm{H} \cdots \mathrm{O}$ interaction in the water dimer are anomalously high for their internuclear distance. It also shows that one better thinks of a hydrogen bond as a strongly interacting triplet of atoms $(\mathrm{O}-\mathrm{H} \cdots \mathrm{O})$ rather than just the $\mathrm{H} \cdots \mathrm{O}$ interaction. Improved insight in hydrogen bonding follows from the nice IQA work on hydrogen bonding[36].

Ángel Martín Pendás. Nice discussion, indeed. Returning to R. G. Parr's "Accurate calculation is not synonymous with useful interpretation" quoted by Patrick Bultinck, are we happy with all the main stream interpretations we teach our students? Let us say, to name just a few, hyperconjugation, the anomeric effect, back-donation,... If we all agree that we need interpretations to jump from calculation (or experiment) to prediction, has the topological approach provided any new chemical insight? I would say yes, and some of the examples in the previous answers show it. I would also like to recall how many concepts used today have been influenced by ideas emanating from the "topological approach": core, lone pair, binding regions (the latter going back to Berlin's work); dihydrogen bonds, halogen (and pcnide, beryllium, etc) bonds; electron localization and delocalization measures, etc. On the other hand, is the present situation idyllic? Here I would say no. I feel, in line with Paul Popelier, that much work needs still to be done.

W.H.Eugen Schwarz. Chemistry is the art and science of less or more complex matter, synthesizable and then really existing under "human conditions", i.e. in the ranges around temperatures of $300 \mathrm{~K} \times 10^{ \pm 1}$, pressures of 1 at $\times 10^{ \pm 3}$ and times of $1 \mathrm{~d} \times 10^{ \pm 6}$. This is a rather special selection of conditions. Accordingly, the laws and ordering rules of chemistry will not be that simple and that general as those of physics, describing idealized material systems in the most comprehensive and general manner. Mathematical chemistry including topological approaches should be useful to find characteristic relations of known descriptors, as well as new descriptors of chemical compounds. There exists a synergic interaction between the synthetic skills of experimental organic, inorganic, macro- and supra-molecular and nano chemists, and the analytic conceptual and computational skills of theoretical chemists of the various breeds. Above, Rzepa mentioned an example.

Many chemical concepts aim at bringing some order into a vast mass of data material. Both the type of compounds and the interests of the investigating chemists vary largely. Accordingly, many useful chemical concepts have been defined in somewhat different ways by different researchers. Examples are atomic radii, bond orders, valence numbers, iconicity and electronegativity, hard- and softness of acids and bases (HSAB), aromaticity, solvent polarity, periodicity of elements, and so on. All these chemical concepts and their definitions are somewhat vague and offer themselves for criticism. Statistical concept-analyses (main-component, factor, cluster analyses) have shown that the various electronegativity and effective atomic charge scales all 'measure' basically the same thing with some noise (meaning a small specific contribution for each individual atom and bond in each molecule or crystal), but using different charge units; i.e. electronegativity is a one-dimensional property[37]. New definitions are still possible, simply enlarging the number of basically similar suggestions. The solvent polarity should be described by two parameters, it is a two-dimensional property [38, 39]. The hardness and the softness are one- and two-dimensional, respectively[40]. The aromaticity concept seems to be three-dimensional[41]. Reducing any of these chemical concepts to a single physical noiseless parameter eliminates some part of the richness of chemistry! I remind that it had been suggested in the QTAIM[42, 43] that chemists should forget much of their experience about different bonding types (covalent attraction, Pauli repulsion, ionic attraction, ionic repulsion, van der Waals attraction, and a new infant that has admittedly not yet shown its chance of survival is the so-called charge-shift bonding) since QTAIM does not allow for such a rich description. However, adding various topological approaches to the toolbox of theoretical and conceptual chemistry, without forbidding the use of other successful concepts, can enrich chemistry with new concepts and throw new light on old ones.

Since "chemistry is creating its own subjects", it may happen that indeed new concepts and new notions are appropriate for new fields of chemistry, concerning new classes of compounds and reactions, or new applications, or new points of view on known compound classes. However, sharpening the definitions in chemistry is somewhat dangerous, owing to two aspects. First, chemical concepts should be applicable to a large number of cases which are all somewhat different. If one confines oneself or even the whole community to a single "sharp and clear" definition, 
one refuses all the other, may be more fruitful options. For instance, the concept of general fuzzy atomic radii for atoms in a given bond state and coordination multiplicity (coordination number) allows predictions of structures, explanations of trends and the discovery of exceptional cases. One may introduce the topological radius of each atom in each molecule in each direction as one of several accurate descriptors for cataloging the chemical compounds. Yet one still needs the fuzzy concepts both in research and teaching[44]. Pupils and young students like simple answers to complex questions. The definition of sharp criteria allows strict qualitative classification of border cases. A most welcome rule, found in some textbooks, reads: A bond is covalent if the two bonded atoms differ in their electronegativities by up to 0.99 ; for electronegativity differences of 1.00 or more the bond is ionic. I strongly oppose against this type of black-white world views, which can be promoted by offering only one sharp criterion for the judgment on complex issues.

Summarizing, new chemical concepts can be found by the topological approaches, provided one follows the dicta of chemical usefulness. Traditional chemical concepts can be supported, enriched, elucidated by topological approaches, again provided the chemical needs are taken as the maxim.

Alexander Sax. I am sceptical about a predictive power of a topological approach, but I see that traditional chemical concepts can be supported by topological approaches. I just mention the recent paper by Arne Lüchow[45], or A. Scemama et al[17], showing that the Lewis electron-pair concept is related to maxima of the squared wave function. Or think of the arrangement of like spins in the valence shell as described by Lennard-Jones[46]. This was found without topological approaches, but it can be nicely visualized using quantum Monte-Carlo methods.

Philip Coppens. Topological theory of the electron density as initially developed by Richard Bader constitutes a new paradigm with a different language from that used up to that time. History is full of examples of new paradigms encountering fierce opposition. But in this case the theory complements existing interpretive methods by adding new concepts, such as the Laplacian, atomic basins bounded by zero flux surfaces, the source function and many others. As Richard emphasized many times, the electron density is an observable. Consequently topological analysis has been widely applied in X-ray Charge Density Analysis and greatly contributed to its results. Yes, new chemical concepts have indeed been found and are utilized by application of the topological approach. They continue to be developed.

Piero Macchi. It could occur. Because chemical concepts are always produced by a particular theoretical model (one for all: "isolobal analogy", coming from fragment molecular orbital theory), a topological approach may also give rise to new concepts. However, one should make sure that concepts are really new, not simply the translation of the old ones in a new language.

\section{What is the status of a chemical concept within a topological approach?}

Does the topological approach yield results concerning well-defined chemical concepts? or Is the output of the topological approach identified with weakly defined chemical concepts?

Paul Ayers. Certainly the topological approach helps clarify and elucidate chemical concepts. I'm not sure about "well-defined" chemical concepts. For example, Richard Bader's work certainly helped clarify the concept of chemical bonding but I don't think the chemical bond is a well-defined concept (and perhaps it never shall be). Similarly, ELF and related approaches help clarify the nature of "lone pairs" but these concepts, alas, are also perhaps not "well defined" (in the sense that not everyone can agree on a definition). So in this sense, I think that the topological approach elucidates (and allows one to quantitatively define, at least in some cases) weakly-defined concepts. Perhaps this is not always true: polarization and polarizability are concepts that everyone can agree on (I think, perhaps I should not be so sanguine). I don't know if polarization has a topological signature but, if it did, then the topological approach could be helpful for both weakly-defined and well-defined chemical concepts. Even so, the topological approach seems to be mainly applied to weakly-defined concepts.

Mauro Causá. The topological approach (QTAIM, ELF and MPD) helps in distinquishing between "strong" and "weak" chemical concept. I define as "weak" a chemical concept that is based only on chemical phenomenology and that poorly resists to a strictly physical analysis. In the next future the topological approach, that is firmly based on physical general concepts, could be used to easily eliminate "weak" chemical concepts. 
Vladimir Tsirelson. The topological approach started from well-defined chemical concepts and it now mainly focuses on "weakly defined chemical concepts". That is a true way. Also, I agree with Paul that approach aims to reach the quantitative level. Let me cite Richards Bader (private conversation in his home, Burlington, January of 1994): "Vladimir, my top achievement is the application of [Schwinger] action principle to bounded atoms. I brought the numbers to chemistry".

Paolo Lazzeretti. The ill-defined concept of aromaticity can be dealt with on the "magnetic criterion", allowing for topological tools, e.g., the "stagnation graph" of the quantum-mechanical current density induced by an external magnetic field. The analysis of the singular points at which the graph lines branch out provides fundamental quantitative information.

Ramon Carbó-Dorca. The topological approach seems to have provided many ways to look at the connection between empirical chemical concepts and theoretical results. However, trying to deepen on such a connection can produce more confusion than new insight, if no proper basis for the new point of view is present. Typical examples of this situation are the chemical bond and the related concept of aromaticity. Has topology ameliorated the visualization of chemical bond by means of Bader analysis, for instance? In many cases one can answer affirmatively, with some exceptions. A new way to look at the density function has arisen from Bader's simple procedure and the resultant graphical results have been printed in innumerable papers. But nothing really new has appeared. Has aromaticity provided insight over molecular structure, or has been just an excuse to publish more of the same? Now aromaticity is like an overdimensioned phantom, which haunts an incredibly vast segment of the literature. The concept has been useful to extend benzene particular chemical behavior to many other, even quite dissimilar structures. Yet, has this changed chemistry in a way to gain effective information about molecules?

Patrick Bultinck. To me a well-defined concept would be an observable or at least something that leaves no room for debate. A horse is a horse and is biologically well-defined. A unicorn is not and I have the impression too often discussions go on and on, discussing whether a unicorn is brown or white. So my opinion is that topology gives excellent insights in chemical concepts but do not provide a stronger definition of the concept. Once accepted that a concept, however weakly defined from a hardcore point of view, might be of interest, topology allows us to understand it better. But when you build a castle on quicksand, even if in the end the castle looks like the strongest one since Camelot, it is still built on quicksand and might sink... However, a too strict stance may result in disconnecting with the majority of chemists who feel comfortable with concepts while at the same time, this same rigorous attitude could also forbid working with present concepts that may in the end result in observables or link very closely to them.

Shant Shahbazian. "Anything" emerging from topological approach is mathematically rigorous and well-defined since the whole procedure has this character. If a concept is ambiguously defined like the "chemical bond", then it has no "unique" analogue in "entities" emerging from topological procedures. In such cases there is no "one-to-one" correspondence between the ambiguously/weakly defined chemical concept and topological indicators. However, it is feasible seeking for loose connections. For instance, topological indices derived from the topological analysis of the QTAIM can be used to classify chemical bonds using “correspondence rules"[47]. Since subjective elements are always involved in constructing correspondence rules, the emerging classifications always have vague "borderlines". Thus, the initial floppiness remains even after "quantification"[48].

Miquel Solà. There is not a unique way to define atomic partitions and all of them have its own limitations. Consequently, when we define a chemical concept within a topological approach the result is always at certain point arbitrary because the outcome is determined by the partition. The degree of arbitrariness depends also on the definition. If we can impose certain physical properties to the quantity defined and the definition proposed obeys these properties, the result can be a reasonably defined chemical concept. This is the case of the recent definition of local spin[49] that provide a way to describe polyradicals in the framework of quantum mechanics. In other cases, the link between the quantities derived from a topological analysis and the chemical definitions is fuzzier and the concept is weakly defined. This is the case of the equivalence between the bond critical point and the chemical bond in the QTAIM theory of Bader et al. Although this point is still controversial, evidences that such relationship is too stringent have been accumulated over the years[50-56]. 
I do not think that aromaticity as a chemical concept is more or less ill-defined that let's say the chemical bond or the similarity between molecules. For some reason, the concept of aromaticity has a bad reputation (probably it comes from the so many existing different possible measures), but there is no doubt that it is necessary to understand the chemistry of many compounds. If you find an alternative way to understand why $\mathrm{C}_{6} \mathrm{H}_{6},\left[\mathrm{Al}_{4}\right]^{2-}$ or $\left[\mathrm{B}_{12} \mathrm{H}_{12}\right]^{2-}$ are particularly stable, then maybe we could abandon the aromaticity concept. It seems to me that we have not reached this point yet.

Simon Grabowsky. Why do we need to justify the use of topological analysis with its connection to chemical concepts? Why is it so important to discuss whether, e.g., the QTAIM bond path and the chemical bond relate to each other closely or not or if a monosynaptic ELF/ELI basin really represents a lone pair? I prefer to make use of the fact that the topological analysis of the electron density or the ELF/ELI indeed gives us numbers (as Richard Bader stated, see Vladimir Tsirelson's comment) for statistical analysis within a physically meaningful framework. If the outcomes of these statistical or correlation analyses help us to control, e.g., substituent effects in a chemical system, the behaviour of materials in different compositions, crystal packing effects, reactivity etc. (and there are numerous examples for successful applications of topological analysis in these different fields[47, 57-69]), then it doesn't really matter how deep the philosophical connection to well-defined or weak chemical concepts is. In other words, we don't need to find justifications for the use of topological analysis if it proves to be helpful for a certain application or chemical question.

Carlo Gatti. To answer this question, we should first ask ourselves what is a "well-defined" and what is a "weaklydefined" chemical concept. I do hardly believe to be able to trace a neat border between the two, nor I believe other may do, despite their, admittedly useful, broad distinction. In particular, a chemical concept may be strong or weak depending on the circumstances. The "chemical bond" is clearly a good example of what I'm trying to say. No one will doubt about the existence of a $\mathrm{C}$-C bond in ethane, although it will be described in different ways by the various topological approaches. However, recent literature is crowded of endless discussions about the existence or not of bonds in more delicate situations. When the chemical concept becomes fuzzy, the topological approach also becomes fuzzy in its answers, but eventually it turns out to be definitely useful and informative. The answer one obtains in such cases, commences to depend on the selected topological approach, on the kind of wave-function, on the "quality" of the basis set. Or, on the experimental side, if we are dealing for instance with topological descriptors based on electron-density derived quantities, the answer starts to depend on how the X-ray data have been sampled, treated, modelled....The topological approach may serve, therefore, as a tool to understand how "weakly defined" is a chemical concept in given circumstances. It provides in those cases a lot of details, which may be useful to quantify, order and classify situations which are by their nature hardly manageable and understandable in other ways. Another interesting chemical concept is that of atomic shell structure. We know that there many suitable functions able to qualitatively recover this structure and to be more or less close to the "ideal" one in terms of number of shells and associated populations. In general, the description of the atomic shell structure by the topological approaches is indeed quite robust. Still this concept, which is "strong" in terms of the principal quantum number, may become loose, hence "weak" in terms of the various functions and related topological approaches, when the shells and the electronic energy levels [typically, $(n+1) s$ and $(n) d$ for transition metal elements] start to be not so clearly differentiated. The fuzziness of the topological approach is such cases just reflects a change in the physics of the problem. Chemical concepts, especially the "well-defined" ones, are quite often the cumulative result of the observation of the chemical behaviour of many classes of systems, but usually under quite "standard" conditions. When applied to more "exotic" situations (high external pressure is a very good case) topological approaches yield results that provide insights about the robustness or not of a chemical concept or of "standard" chemical behaviour in conditions far out from equilibrium. In such cases, new chemical concepts may be revealed by the topological approaches. For, instance, a simple example is provided by the unexpected partially ionic behaviour of an element (B) under pressure[70].

Paul Popelier. First, a short reaction (rather than answer) to the subquestions. Science should strive for the crispest possible definition of a concept, especially when given enough time and especially in Chemistry, which studies a complicated universe. In the long term I am therefore uncomfortable with "weakly defined" concepts, which could be an excuse to do poor science, if no-one tries to strengthen the definition. I am not trying to oversimplify the chemical universe but I defend clear thinking. If one tries hard enough "weakly defined chemical concepts" should eventually disappear (see also last part of my answer to Question 6). One of the prime outcomes of QTAIM is a modern, 
computable and practical definition of an atom inside a system. Our work on a protein force field with multipolar electrostatics now explores the addition of intra-atomic energies. Tests on oligopeptides show that this atomic energy ("self energy", which includes kinetic, Coulomb and exchange energy) for a carbon atom in tri-alanine, for example, changes by only $0.6 \mathrm{kJmol}^{-1}$ compared to the same carbon energy in penta-alanine $\left(0.4 \mathrm{kJmol}^{-1}\right.$ for isoleucine and threonine). This high degree of transferability shows that a topological atom is a useful high-information fragment carrying a precise energetic fingerprint of its chemical environment. At the same time, this excellent energetic transferability shields the force field from the huge (non-chemical) energy released when an atom is formed from its bare nucleus and electrons. As such, the topological atom forms a bridge between the physical Hamiltonian (written in terms of non-chemical entities that are nuclei and electrons) and chemical entities (that are atoms). Topological atoms are fragments that have unique kinetic energies[71] provided one stays within the "Laplacian family of local kinetic energies"[5]. Secondly, the topological approach offers a computable yet intuitive picture of chemical bonding but this picture is not yet complete. Historically[11], QTAIM first defined the atomic energy and then, many years later, the bond critical point. The meaning of the latter is still not fully established but a promising approach, in my opinion, is linking it with energy partitioning, as pioneered[72] in 2007 and further worked out more recently[73].

Ángel Martín Pendás. Are there well-defined concepts in chemistry? Maybe we should start with this. An observable (Patrick) is well-defined, but is it a chemical concept? How many quantum-mechanical observables may be associated to chemical concepts? I am afraid that the number is small, to be optimistic. Ordinary chemical thinking is plagued with fuzzy concepts and ideas. From this point of view, attempts to give them a solid foundation should be gauged using standard philosophical rules: minimality and closeness to the physical models which we believe rule the world (i.e. quantum mechanics). In my opinion, topological schemes approach these rules better than, for instance, orbital based reasoning.

Alexander Sax. Weakly defined chemical concepts are only obtained when the weak topology is used.

W. H. Eugen Schwarz. In the formative period of the chemical science, say before the late 18th century, chemistry was a mainly qualitative science, with hardly any serious conservation law. (I may say so, although the synthetic prescriptions contained mass and volume instructions, and although 'recycling' substances such as sulfur or copper through what we now call oxidation and reduction were rated as important empirical evidences for the early chemical theories.[74]) After the creation of the modern chemical science by the group around Lavoisier in Paris, a positivistic period set in putting emphasis on numerical weight relations and strict connectivity relations, i.e. on numbers and topology. Developing intuitive explanations with the help of hypotheses had a hard time. During the second half of the 19th century, the development of thermodynamics, statistical mechanics and molecular mechanics, and half a century later of quantum chemistry gave both, numbers and the first steps towards a causal understanding. Yet large parts of the chemical community believe mainly in positive numbers, so that from time to time a minority cry can be heard: don't give me (chemical) numbers, give me (physical) insight.

Concepts are created by scholars, thus the status of chemical concepts is determined by the personality and the philosophical (pre)conceptions of these various individuals. Anyhow, typical chemical concepts throughout have two different aspects, a quantitative hard side and a qualitative soft, fuzzy side. Geometric structure may be specified by the numerical geometric parameters of the (quantum and thermally smeared) positions of the atomic nuclei or qualitatively specifying the connectivity of the atomic core regions. Energetic stability may be accurately specified by the numerical thermodynamic parameter set or qualitatively through the bond strengths or bond orders. Chemical compounds are assumed to consist of conserved atoms hold more or less permanently together by bonds. The atoms can be specified by their rather constant averaged isotopic weights, or by their rather variable spatial and charge distributions. Concerning the numerical quantitative aspects of chemical concepts, probably the topological approaches can't contribute more. However topological approaches can become relevant for the qualitative weak fuzzy side of the chemical concepts.

If topological approaches can contribute some numerical characterization indices, one may first check correlations with other numerical parameters suggested in the same context. Main-component analysis can elucidate whether the topological parameter(s) corroborate previous chemical views or add some new aspect. The discussion in the chemical community will, hopefully after some years, come to a conclusion, which numerical parameters are most fruitful for the chemical concept with a given name, and whether such a concept is linear one-dimensional and may be described 
by a real number, or multidimensional to be describable by a point in a two- or three-dimensional parameter space (such as polar solvent, basic ligand, aromatic molecule).

All chemical concepts are quantitative (numerical in some meaning parameter space), even if sounding qualitative (yes-no, more or less: polar - covalent or aromatic - non-aromatic - anti-aromatic). There is a tendency to classify chemical substances, chemical groups, chemical reactions etc. This is fine for getting a first overview and for introductory teaching, and it works well for the clear cut cases. Indices which are integer or yes-no are good for some book-keeping. E.g. whether $\mathrm{C}$ is counted as tri- or tetra-valent in the $\mathrm{C}_{2}$ molecule is not that relevant, what matters are its spectroscopic and reactive properties. Topological indices that are of this discrete type are nice for the clear-cut cases. Examples are the synapticity of an ELF region, the existence of an internuclear bond path or a non-nuclear electron density maximum. Changing the molecular system a little, or changing the computational or experimental approach a little, may change the topological index from two to one, or from one to zero. It makes little sense in such border cases to define a numerically unstable criterion as a chemically relevant characterization.

An example is the appearance of the "non-nuclear attractors" in the molecular and crystal electron density distribution. Atomic matter follows wave mechanics. Localized states show a wavy exponentially decaying density. If only a few states are occupied (as in light or electron-deficient atoms) the wavy nature of the density can be recovered more or less easily. Local perturbations of a rather homogeneous electron gas induce the Friedel oscillations. If more states with their density maxima at different positions are superimposed, it becomes more and more difficult to recover density wave maxima. This is the case for heavy atoms: light atoms show an electron density shell structure, heavy atoms only show the shell structure in the cores, no longer in the valence region. As Martin et al.[30] have shown, non-nuclear electron density maxima are nearly ubiquitous though not for all internuclear distances. They appear more or less accidentally and seem to have no specific meaning when they just show up at equilibrium bond lengths. Similarly, there may occur bond paths in the electron density distribution sometimes rather accidentally, indicating the near-formation of a bond, or just a non-bonded contact, looking from various chemical points of view[75, 76].

Summarizing, the yardstick for chemical concepts is practical chemistry and the ordering desire for the vast mass of phenomena. Chemical concepts can appear in two forms, as physically well defined, quantitative numerical ones, and that's it. Or as 'typically chemical', soft, fuzzy ones. Here topological results, both of qualitative and quantitative kind, can help on the way of clarifying the meaning, always keeping the chemical purpuses in mind.

Piero Macchi. The question is difficult to answer. A chemical concept may be fully embedded in the topological approach or it may cross two or more theoretical frameworks. Therefore its status may substantially vary.

The most classical example is the chemical bond. While it is in principle a standalone concept within topological methods, it normally embraces several approaches. This makes it not perfectly compatible with all interpretations, but more general and universally understood. A concept like the bond order, instead, cannot be defined by the "classical" quantum theory of atoms in molecules, although some indices may well reflect this concept. However this is an example of the "translation" of a concept defined within molecular orbital theory into the framework of topological approaches.

Sometime, concepts defined by two approaches are quite unrelated or even conflicting. For example, the stability of a molecular graph is clearly defined in topology, but it has little analogy with other approaches and it has nothing to do with the stability of a molecule, which is what the typical reader of chemical journals would understand.

\section{Should topological approaches provide measurable quantities?}

Paul Ayers. Yes. Ideally we should base our understanding on quantities that are, at least in principle, measurable. Doing so forces a certain intellectual discipline, and keeps one discussions grounded in reality. But this is not always practical; I would not wish to discard all of the (sometimes very useful) exceptions to this rule. E.g., the topology of Hartree-Fock/Kohn-Sham orbitals is obviously very useful, even though these orbitals are manifestly unobservable.

Mauro Causá. Yes. Topological analysis will provide a quantitative measure of the chemical hypotheses. This target will be reached for example using the ELF critical branching values or the MPD values of an electronic structure.

Vladimir Tsirelson. Yes, I think so. 
Paolo Lazzeretti. Hopefully, yes.

Ramon Carbó-Dorca. I'm afraid to slightly disagree with the previous comments, if there is not added some nuance into the question. If the basis of the topological approach is quantum mechanical, usually in terms of a density function, then the only way to provide measurable, observable information is to define topological results by means of a suitable hermitian operator acting on the density. Visualization of density features might be loosely connected by such a claim. However aromaticity hardly meets such a condition.

David L. Cooper. The chemical concepts that continue to prove to be the most useful tend to be those that allow us to rationalize important features of e.g. molecular properties and reactivity. Much as we might prefer to concentrate on deriving measurable quantities from topological approaches, the reality is that some of the most useful chemical concepts that have stood the test of time are far from easy to quantify. As such, it seems to me that it would be somewhat limiting simply to reject outputs from topological approaches that are more qualitative, such as pictorial representations of molecular electronic structure, for example.

Patrick Bultinck. As Paul W. Ayers says, we need intellectual discipline. As David L. Cooper implies: if we now all of a sudden reject all age-old concepts that have guided and are guiding e.g., organic chemists in synthesis, we'll be fools to think this will be accepted anytime soon. I do like the intellectual discipline and for me this also means that we keep reminding ourselves that these concepts were never meant to be used as quantitatively as they are now. There are no heaven-sent atoms in molecules and it is pointless to claim the atomic charge is too large or too small with method $\mathrm{x}, \mathrm{y}$ or $\mathrm{z}$. More effort should be devoted to at least understanding why the result is what it is. And what if we base all our analysis on observables ? What with e.g., the ever so interesting underlying matrices as in density/density matrix, property/property density matrix, ... What about interesting functions that -in my limited knowledge- are not (yet) truly observable like the current density in an aromatic molecule ? As Paolo Lazzeretti wrote in his paper on aromaticity based on observables: Electronic currents are not directly observable, but are related via integral formulae to the magnetic properties[77]. This suggests that we need to leave some freedom. Current densities are not observables but they are key in the Biot-Savart law that ultimately is key to NMR and thus current densities lead to observables in a pretty direct way. So to conclude; maybe not all should be based on strict observables (as Ramon Carbó-Dorca says: expectation values from Hermitian operators) but we need to be very cautious when we might drift too far away from observables too.

Shant Shahbazian. The concept of measurable quantity (probably means "observable" here) is more problematic that is usually assumed[25, 78]. So, the answer is an explicit "no". Topological approach is a mathematical procedure that can be used to decipher topological characteristics of both 3D scalar functions that are usually assumed to be measurable like charge densities as well as those that are assumed to have purely mathematical origin (though, personally, I do not defend this distinction). What is really important is whether a topological procedure is effective enough to reveal characteristics that are "correlated" with chemical experiences. If yes, then the proposed topological procedure is successful in its mission.

Miquel Solà. Ideally yes, but the problem is that any chemical concept defined within a topological approach gives arbitrary results because the outcome depends on the partition and there is neither a unique way to define atomic partitions nor a manner to decide which partition is better. By comparing the results obtained with the "chemically" predicted results, in some cases it is possible to conclude that certain partition does a better job than others. For instance, calculation of bond orders with the Mayer and fuzzy atom approaches are closer to the formally expected bond orders than QTAIM delocalization indices[79].

Simon Grabowsky. No, topological approaches definitely do not need to provide measurable quantities. And again, as with the previous points, I closely agree with Shant Shahbazian. As an example, the great strength of the topological approach is to be able to quantify bonding phenomena, which are inherently not measurable, and yet it provides a deeper understanding of how bonding manifests itself on the sub-atomic electronic level than most experiments can. 
Carlo Gatti. The electron density and related functions (e.g. its Laplacian) are observables (as properly defined by Dirac and reminded by Ramon) and with some proviso also "measurable" quantities. Topological approaches reveal values at and locations of their critical points, hence measurable quantities. But I do agree with David's view that topological approaches should not be constrained to only such kind of quantities. For instance, one may use topological approaches, applied to measurable quantities like the electron density and related functions, to assign approximate values to the relative weights of the resonant forms (VB structures) describing a system and to judge how such weights are slightly modified by a change of phase (e.g. from in vacuo to crystal) or of a substituent. Although resonant forms and their weights clearly represent an hypothetical and idealized view of a system and all but measurable quantities, they still provide tremendous chemical insight. Another example is that related to the value of the dipole moment of a molecule in crystalline phase. Topological approaches (QTAIM) may provide this value and also its often recovered magnitude increase upon crystallization. The molecular dipole moment in condensed phase is not a measurable quantity, but still a very useful one for quantifying the importance of packing effects and, in general, the changes of the properties of a molecule when crystallized.

Jerzy Cioslowski. One has to be careful about the definition of measurable quantities. In principle, anything that is derivable from the electronic wavefunction alone (i.e. derivable through a well-defined sequence of mathematical operations that does not vary from one molecule to another and does not involve additional input) is measurable as experimental measurement of electron density and energy suffices to define the electronic Hamiltonian and identifies the electronic state. Within this interpretation, even the exact KS orbitals are measurable as they can be obtained through a well-defined mathematical manipulation of the wavefunction. On the other hand, any quantity employing basis sets (such as the Mulliken charges) is not measurable as it relies on an additional input of basis functions. I guess one should not frown upon such generalization as even the "honestly measurable" quantities such as electron density are in fact not measured directly but derived from other data such as diffraction intensities.

Paul Popelier. Measurement is a concept that can be stretched. Originally, it refers to what our human senses can detect directly. Humans were able to expand their observable universe to see, for example, images of UV light or hear ultrasonic sounds (which some animals can do naturally), and of course measure much more (see Herschel telescope). "Enhanced measurement" can also occur on computed data. For example, a molecular dynamics simulation of a crystal nucleation event in solution offers data that no experiment can deliver. The set of system snapshots and their properties then become observable. However, a major concern is that this simulation must be (sufficiently) realistic. In other words, it must map onto reality in an accurate way. If not, interpretation will derail and Frenking's unicorns[80] will appear. If the mapping to reality fails then one will make false observations. All this also applies to a theory: if a theory maps onto reality accurately then the concepts this theory proposes also become part of reality. In that sense, Newton's laws are real, and when pushed further, even the imaginary number i is real (no pun intended). So, practically, this means that the topological approach needs to prove continuously that it maps onto observed reality as accurately as possible. While doing so it creates, within its bosom, other observable quantities (in the enhanced way described above). Carlo's example of a molecular dipole moment in the condensed phase nicely illustrates this assertion.

Philip Coppens. Not necessarily. Theory is often used to extend experimental observations to obtain information of importance that is not directly accessible to experimentation but provides further insight. With some exotic exceptions orbitals can not be directly measured and can be defined in many different ways. But if an atomic connectivity and structure are accurately known much information can be gained by now classical theoretical calculations, information which may not be directly accessible otherwise. The same is true for topological analysis.

W.H.Eugen Schwarz. Yes, topological approaches should provide both numerical and qualitative graphical data, always furnished with the caveat that chemical compatibility and usefulness is granted.

Piero Macchi. Any approach that explains chemistry is intended to justify some observations. Therefore a connection with measurements is inherent. 


\section{Is it possible to predict the outcome of a topological approach without performing a calculation on a computer?}

Paul Ayers. I think that most experienced scientists know, before they perform a calculation, about what to expect. So I think that often we can predict the answer before we do the calculation, and often a back-of-the-envelope calculation suffices. However, there are interesting cases where calculations, or even experiments, are helpful: think about using accurately computed, or even experimentally measured, (spin-) electron densities to elucidate the bonding pattern in complex materials. In those cases, the experiment/computation is done because we don't know what to expect.

Mauro Causà. A full knowledge of the results of a topological analysis can be reached only performing calculations or measurements. QTAIM needs the detailed knowledge of electronic density, that in many cases can be reached. ELF is not directly an observable, but is possible to fit at observed density laplacian to a model local kinetic term. MPD at $n$ level could be derived in principle from experimental reduced density of order $n$. On the other hand is possible to think about a moderate transferabilty of topological quantities.

Vladimir Tsirelson. No. Unfortunately no. Of course the experienced worker certainly knows what he/she can expect from calculations. However our fixed belief can play a spiteful joke...

Paolo Lazzeretti. Although a guess is sometimes possible, a thorough computational investigation is frequently needed.

David L. Cooper. As well as helping with the rationalization of important features of e.g. molecular properties and reactivity, we should seek outcomes of topological approaches that also allow useful predictions to be made for more complex systems, preferably without the need to perform a full calculation for every system of potential interest. Often, though, the results that are really interesting are those for which the outcome of the topological approach are somewhat different from those that we anticipated.

Bernard Silvi. As far as qualitative or semi-quantitative chemically related properties are concerned the answer is certainly yes. I see two reasons for that. On the one hand the axioms and the theorems of the topological model constrain the output. On the other hand, Chemistry proposes a series of very efficient tools such as the Periodic Table, the octet rule, electronegativity scales, Lewis's model, VSEPR rules which enable (together with chemical knowledge) to anticipate the trends of the outcomes of a topological approach. Moreover, at least for QTAIM and ELF, a moderate transferability is observed justifying cut and paste strategies as first steps of a study.

Patrick Bultinck. I think there is room for debate here. For a method to be useful, it is best if the outcome can be roughly predicted using the back of an envelope. This is what most chemists are used to (and frankly, willing to do). For theoreticians, as David L. Cooper says, it is interesting to study cases where the outcome is a but unexpected from what was anticipated or appeared from the back of an envelope, but most chemists will not expect such accuracy in a prediction.

Shant Shahbazian. The general answer to this question is "no". Topological approach is usually used within context of theories that aim to extract chemical information from intricate ab initio wavefunctions not from "model" simplified wavefunctions derived from "paper and pencil" methods (like Hückel's approach). However, it is probable that for an "expert" eye it is feasible to guess (but not predict!) the outcome a topological analysis with a high degree of confidence. But, there are always "surprises" even for experts that exotic Molecular Graphs emerging occasionally from the topological analysis of the QTAIM are the best examples. If a "paper and pencil" method is capable to predict the outcome of a topological procedure, then what would be the motivation to introduce the procedure per se?

Julia Contreras-Garcia. I think that most of us know the answer to common systems without calculating it. What I would say is even more interesting is that we can identify to which systems we know the answer, and for which ones our intuition could fail. 
Simon Grabowsky. Let me try to answer the question with a short story. During my $\mathrm{PhD}$ work with Prof. Luger in Berlin, we investigated the degree of transferability of bond-topological and atomic properties derived from QTAIM between different organic systems. After a while, we started a competition between the PhD students: Can we guess the values of the electron density at the bond critical points and the atomic charges of a dipeptide within the experimental uncertainties of the measured electron density? Of course, all of us could, because there is no unexpected bonding situation in such simple organic systems. So we just needed some experience with the topological analysis of similar systems to predict the values as accurately as a measurement can give them. Consequently, we asked ourselves why we were actually pursuing these analyses if we don't learn new things about chemistry? The answer was that we were developing library methods based on transferability assumptions that can quickly and reliably reconstruct an electron density from building blocks in order to get better experimental geometries and derived properties, such as interaction energies, that cannot be guessed[81, 82]. However, in my opinion performing topological analysis of compounds in usual bonding situations in the ground state is not more than collecting stamps, but the interesting applications involve method development and chemical problems.

Carlo Gatti. I always suggest my students to guess (or roughly "predict") the outcome they expect before making a calculation. This holds true also when they make use of a topological approach. And I'm clearly obliged to do the same, myself! Besides being the only way to easily uncover possible computational mistakes, this attitude helps to understand the outcome, to appreciate its smaller or larger deviation from the guessed one, and even more importantly, to be able to recognize a possibly unexpected result. Which, as it has been already said by many of you, is clearly one of the main reasons motivating the use of topological approaches and of why we keep have fun working with them... That is to exploit a multi-facets method which in usual conditions recovers what is already known, boring and expected, but that becomes really insightful and sometimes definitely surprising when the standard chemical paradigms fail or become uncertain or inapplicable.

Jerzy Cioslowski. Fortunately, it is usually not possible to predict the outcome of calculations with absolute certainty. I write "fortunately" because this fact keeps us employed (and comfortably so...).

Paul Popelier. My answer is yes but then one should question the value of such a prediction because a back-ofthe-envelope prediction is only coarse. An example from recent work[35] shows that the exchange energy between two hydrogens in a $[\mathrm{H}-\mathrm{C} \ldots \mathrm{C}-\mathrm{H}]$ system in saturated hydrocarbons is consistently higher if this four-atom system is planar. This phenomenon clearly appears in a plot of exchange energy versus H...H internuclear distance, in the set of ethane, propane, butane and pentane. Without calculating the case of hexane one predicts correctly that, also here, the planar configuration will again yield a larger H...H exchange energy than in a non-planar configuration, for the same internuclear distance. This case study delivered a clear trend and hence a rule. This rule can be applied to cases outside the original set of observations and hence make a prediction without computation. However, the topological approach should be used more at the cutting edge of Chemistry, where open discussions occur that can decide on the performance and even validity of topological versus non-topological methods.

Ángel Martín Pendás. I think that we all agree that no scientific field acquires that status until it becomes systematized. This means that someone was able to find order in the raw data. Chemistry is a successful, predictive science, so anyone approaching it from the topological side should be able to "predict" qualitative- or semi-quantitatively the outcome of a calculation beforehand in those cases that were used to forge the field. And no one should be able to make such a prediction in what we today think as interesting systems. What is important is that the border between those two sets moves in time to hold more and more boring (predictable without calculation) molecules as we improve the topological machinery at our disposal.

W. H. Eugen Schwarz. First there are the computational as well as the mathematical and paper-pencil approaches. Concerning the second ones, most questions can be directly answered, though so to say at the level of the Hueckel approximation. Concerning computational, e.g. QTAIM or ELF investigations, on the other hand, an experienced scientist should have a good feeling for the results, and should mistrust the computation if it deviates strongly. Of course there are also the 'special cases' and the 'borderline cases', and there the results cannot be guessed but can supply arguments, e.g. is there a bond, is there a multiple bond, what is the shape of the lone pair. 
Piero Macchi. Yes, definitely. Calculations may prove or dismiss hypotheses, but they do not create themselves hypotheses.

\section{What are new domains for which topological approaches would be useful?}

Paul Ayers. I feel that topological approaches have been broadly used as a tool for understanding chemical phenomena, but not as much for actually making predictions. There are a few exceptions. For example, Samantha Jenkins has found "missing topologies" for clusters and used that to identify new stable cluster geometries. In the zeolite community, they're making an exhaustive (or at least nearly exhaustive) search for structures guided by "what is topologically possible." But shouldn't topology also be useful in more practical realms? Can topology be used to design an appropriate catalyst? To screen possible drug molecules? I would like to believe that our tools can be used in this way, but there is scant evidence to support my faith.

Mauro Causà. The topological approaches (QTAIM, ELF and MPD) can be useful to completely re-write the general chemistry: that will be useful for complete the synthesis between phenomenological chemistry and molecular physics, initiated by Linus Pauling. The topological analysis of the excited states is, to my knowledge, a totally unexplored field: then topology could define new chemical descriptors, and may be become predictive. The other chemical topology, as the topological analysis of bond networks, could be really predictive.

Vladimir Tsirelson. I can indicate at least two new domains for which topological approach would be useful. These are its combinations with i)DFT and ii) information theory. I personally expect the wave of new results along these scientific directions.

Paolo Lazzeretti. I am presently working on a "falsifiable" (in the Popper jargoon) definition of "delocalized current density" induced by a uniform magnetic field in cyclic and non cyclic molecules. Tentatively, such a current density would be defined as that flowing beyond a topological surface called "separatrix", which separates the internal set of branching points from a nodeless external portion.

Ramon Carbó-Dorca. My experience is very limited, but I will guess that for the moment these approaches have been useful to develop several graphical techniques which might serve to illustrate the molecular electronic entourage, sometimes with beautiful pictures.

Patrick Bultinck. Property predictions (as in some improved QSAR), predicting reaction outcome. Rewriting general chemistry would be, indeed, most exciting but at least for me, that requires first sorting out loose ends. As I stated earlier: QTAIM is a chemically guided topology that at least in two stages has chemical input. 1) concentrate on critical points not associated to nuclei to define AIM and 2) reducing a zero integral condition to a condition of zero integrands over the AIM boundary.

Shant Shahbazian. I am personally working on the extension of the QTAIM beyond the Born-Oppenhiemer paradigm as well as for exotic species[83], I must emphasize that concomitant topological analysis is central to decipher "atoms in molecules" (AIM) structure in these domains. Traditionally, these domains were not considered to be amenable to AIM analysis so the topological approach of the extended QTAIM (termed multi-component QTAIM) is a novel application of the topological analysis that unifies the AIM concept of a large (and traditionally irrelevant) classes of systems (for a recent example see: [84]. Time-dependent topological analysis is another area that may have a bright future when considering molecular dynamics and time-dependent Schrödinger equation for chemical reactions, even beyond chemistry are foreseeable (for some interesting examples of nonorthodox applications of the ELF analysis see: $[85,86]$.

Simon Grabowsky. I believe that the various numerical descriptors and indicators that arise from topological analysis can be used to learn more about reactivity properties if analysed along pseudo-reaction pathways. Polo, Silvi et al. have done some very inspiring work in this resepct using the ELF[87, 88]. If these ideas can be combined with crystallographic experiments in the tradition of Bürgi's structure correlation philosophy[38], we can expect a deeper insight into reactive properties of simple molecules. 
Julia Contreras-Garcia. I think there is one vast field in which topological approaches could have important consequencies: inverse design. One example is materials. Understanding the microscopic reason behind interesting properties is a straight way towards imaterial design. Another nice example is drugs. I think that one of the greatest potentialities of topology is that it focuses on local properties, so that if we were able to make the link topologyproperty/energy, we would not need to worry for the size of the system since we would just care for the local part of the protein-ligand interaction.

Carlo Gatti. I'm working on the extension of the source function concept to electron spin densities, which, should eventually help in elucidating the mechanisms of the transmission of electron spin information (through bond, through space, with ferro or anti-ferro "coupling"... ) in complex molecular systems and materials. I've presented preliminary results at a Gordon Research conference last year and I've shown as an interesting side-product, the rich information one obtains from the Laplacian of the spin density. My work is by now only on calculated spin densities, but due to recent development of spin-split multipole model refinement procedures[89] it will be hopefully possible to extend it also to experimentally measured spin densities. On another side, it is well known that computing electron spin densities for some systems, like the transition metal complexes containing non-innocent ligands, may be sometimes a difficult task, with results that may largely depend on the adopted computational method[90]. Use of a topological approach on spin densities and perhaps also of the source function concept extended to spin densities, might concur in shedding light on such a large dependency. Moreover, although it is not a new domain, but just a recent one, I believe that topological approaches will be still incredibly useful for understanding the unexpected new compounds, along with their exotic bonding patterns that may be formed under high pressure. Rationalizing their observed phase changes with pressure using topological approaches is also quite exciting.

Jerzy Cioslowski. Some time ago, Paul Mezey proposed partitioning of the space of internal coordinates of a molecule into catchment regions[91] that is analogous to the partitioning of the Cartesian space into atoms in QTAIM. Within the Born-Oppenheimer approximation, each catchment region defines a distinct chemical species. In my opinion, this very interesting idea has not been given enough attention by the chemical community.

Paul Popelier. I can think of two big areas where the topological approach could make a difference that will be appreciated by a large community of chemists and material scientists. One big area is understanding in local terms how matter assembles, whether a molecular crystal or a protein-ligand interaction. A topological energy partitioning can detect and characterise hydrogen bonding in its widest sense but also pinpoint other interactions that have no name yet. Much of Chemistry has become an "assembly science" and this is where much systematic work must be done. Secondly, the other big area is that the interpretation and rationalisation of traditional chemistry, as a "single-molecule science", which needs to be revisited and "cleaned up". For example, textbooks do not explain satisfactorily[92] why the $\mathrm{B}-\mathrm{F}$ bond length is so short in $\mathrm{BF}_{3}$. A minimally constructed topological electronegativity scale (without the assumptions[93] of the Electronegativity Equalisation Method or any other framework), is also highly desirable.

Finally, there are two types of research activity we should see more of. There should be more bottom-up research, i.e. apply a topological quantity to a huge number of cases, observe trends and formulate a rule or concept. This is how concepts and rules (old or novel) emerge naturally and rigorously connect to an underlying quantum reality. In contrast, an example of top-down research is: here is a very old and vague concept called aromaticity that needs to be quantified topologically (in spite of its definition changing every 30 years). If aromaticity ultimately boils down to mere "benzenicity" then research should focus on a bottom-up strategy, looking for emerging "patterns of remarkable delocalisation stability", without worrying if those patterns coincide with aromaticity. Give these patterns/concepts/rules new names and use them with confidence.

The second type of research activity is falsification. There are only a few comparative studies[36, 94, 95] between topological and non-topological approaches but the full consequence of a fundamental difference in interpretation between them has not been followed up. For example, in $\mathrm{B}_{2} \mathrm{H}_{6}$, the value of the topological exchange energy between the bridging hydrogens is about three times larger than that between $\mathrm{B}$ and $\mathrm{B}$. In a private communication Roald Hoffmann states "... I noted the HH interaction between the bridging atoms in diborane, this is something new to me; that there is some BB bonding is easier to understand." Here is a clash between a topological method and a non-topological one. Which one is right? Or is a falsehood simply complementary a truth? 
Ángel Martín Pendás. I would add to Carlo on pressure. Without any new tools, the topological approaches differ substantially from other interpretive tools at hand in providing answers wherever preconceptions do not work. Our naïve chemical rules do only apply to a very narrow temperature and pressure regime amenable to experimentation. The rest is terra incognita. Finding new chemical rules valid in extreme thermodynamic conditions (think of planetary science, for instance) may well become a golden territory for topological thinking.

Philip Coppens. Molecular processes in chemistry and biology are now becoming accessible on timescales down to tenths of femtoseconds as a result of the dramatic increasing power and time-resolution of light sources, which is continuing. This is a field that to my knowledge has not been addressed by topological examination. How does the molecular topology change during the early stages of a chemical reaction? Can dynamic changes in the topology of the electron density provide insight in the course of a chemical reaction? How does it change at conical intersections? Can time-dependent topology be developed?

W. H. Eugen Schwarz. The chemical topological approaches have so far largely been limited to the investigation of stationary systems. But chemistry is 'changing the substances', by nuclear rearrangements (chemical reactions) or by electronic rearrangements (photochemistry, electronic excitation). Therefore one open field of development is the investigation of the path from the initial to the final state, with two aims of the research: i) describing the reaction path, ii) understanding, i.e. explaining, why the stationary product has this electronic-geometric structure.

Piero Macchi. Topological approaches are useful not only to rationalize the electron density distribution but in many other areas of chemistry, in particular structural chemistry. Among the best applications are the classifications of extended solids, the predictions of conversion paths in solid state reactions, the rationalization of bulk properties in crystals. It is difficult to anticipate which domain will take more advantage or in which domain the topological approaches will produce more success. Often this depends on the ability of the scientists who apply them to communicate new findings and how rapidly these are received by the scientific community.

\section{Acknowledgements}

Andreas Savin and Bernard Silvi. thanks the editors and the participants of this strange object.

\section{References}

[1] S. Shaik, H. S. Rzepa, R. Hoffmann, One molecule, two atoms, three views, four bonds?, Angew. Chem. Int. Ed. Engl. 52 (2013) $3020-3033$.

[2] E. V. Babaev, Intuitive chemical topology concepts, in: D. Bonchev, R. Rouvray (Eds.), Chemical Topology: Introduction and Fundamentals, Gordon and Breach, Reading, 1999, pp. 167-264.

[3] J. A. Cramers, K. S. Booksh, Chaos theory in chemistry and chemometrics: a review, Journal of Chemiometrics 20 (2006) $447-454$.

[4] K. Ruedenberg, The physical nature of the chemical bond, Rev. Mod. Phys. 34 (1962) 326-376.

[5] J. S. M. Anderson, P. W. Ayers, J. I. Rodriguez Hernandez, How ambiguous is the local kinetic energy?, The Journal of Physical Chemistry A 114 (33) (2010) 8884-8895.

[6] R. F. W. Bader, P. M. Beddall, Virial field relationship for molecular charge distributions and the spatial partitioning of molecular properties, J. Chem. Phys. 56 (1972) 3320-3329.

[7] P. Cassam-Chenaï, D. Jayatilaka, Some fundamental problems with zero flux partitioning of electron densities, Theor. Chim. Acta (Berlin) 105 (2001) 213-218.

[8] R. F. W. Bader, P. M. Beddall, J. Peslak, Jr., Theoretical development of a virial relationship for spatially defined fragments of molecular systems, J. Chem. Phys. 58 (1973) 557-566.

[9] M. Causà, A. Savin, B. Silvi, Atoms and bonds in molecules and chemical explanations, Foundation of Chemistry 13 (2014) $2-26$.

[10] P. L. A. Popelier, Solving the Schroedinger Equation: Has Everything Been Tried?, Imperial College Press, London, 2011.

[11] P. L. A. Popelier, The quantum theory of atoms in molecules, in: G. Frenking, S. Shaik (Eds.), The Nature of the Chemical Bond Revisited, Wiley-VCH, Weinheim, 2014, Ch. 8, pp. 271-308.

[12] P. L. A. Popelier, F. Aicken, Atomic properties of amino acids: Computed atom types as a guide for future force-field design, ChemPhysChem 4 (2003) 824-829.

[13] P. L. A. Popelier, Quantum chemical topology: on bonds and potentials. in structure and bonding, in: D.J.Wales (Ed.), Structure and Bonding. Intermolecular Forces and Clusters, Vol. 115, Springer, Heidelberg, 2005, pp. 1-56.

[14] A. Scemama, P. Chaquin, M. Caffarel, Electron pair localization function: A practical tool to visualize electron localization in molecules from quantum monte carlo data, J. Chem. Phys. 121 (2004) 1725-1735.

[15] A. Scemama, M. Caffarel, R. Chaudret, J.-P. Piquemal, Electron pair localization function (eplf) for density functional theory and ab initio wave function-based methods: A new tool for chemical interpretation, J. Chem. Theory Comput. 7 (2011) 618-624. 
[16] A. Savin, Probability distributions and valence shells in atoms, in: K. D. Sen (Ed.), Reviews of Modern Quantum Chemistry: A Celebration of the Contributions of Robert G.Parr, Vol. I, World Scientific, Singapore, 2002, pp. 43-62.

[17] A. Scemama, M. Caffarel, A. Savin, Maximum probability domains from quantum monte carlo calculations, J. Comput. Chem. 28 (2007) 442.

[18] C. S. M. Allan, H. S. Rzepa, Chiral aromaticities. AIM and ELF critical point and nics magnetic analyses of mobius-type aromaticity and homoaromaticity in lemniscular annulenes and hexaphyrins, J. Org. Chem. 73 (17) (2008) 6615-6622.

[19] H. S. Rzepa, The chiro-optical properties of a lemniscular octaphyrin, Org. Lett. 11 (2009) 3088-3091.

[20] S. M. Rappaport, H. S. Rzepa, Intrinsically chiral aromaticity. rules incorporating linking number, twist, and writhe for higher-twist möbius annulenes, J. Am. Chem. Soc. 130 (2008) 7613-7619.

[21] R. J. Boyd, K. E. Edgecombe, Atomic and group electronegativities from the electron-density distributions of molecules, Journal of the American Chemical Society 110 (1988) 4182-4186.

[22] R. J. Boyd, S. L. Boyd, Group electronegativities from the bond critical point model, Journal of the American Chemical Society 114 (5) (1992) 1652-1655.

[23] R. S. Mulliken, Molecular scientists and molecular science: Some reminiscences, J. Chem. Phys. 43 (1965) S2-S11.

[24] R. G. Parr, Density-functional theory in chemistry, in: R. M. Dreizler, J. da Providencia (Eds.), Density Functional Methods in Physics, NATO ASI B Series, Plenum, New York, 1985, p. 141.

[25] S. Shahbazian, Letter to the editor: Are there "really" atoms in molecules?, Foundations of Chemistry 16 (1) (2014) 77-84.

[26] U. Koch, P. L. A. Popelier, Characterization of c-h-o hydrogen bonds on the basis of the charge density, J. Phys. Chem. 99 (1995) 9747-9754.

[27] C. Gatti, F. Cargnoni, L. Bertini, Chemical information from the source function, J. Comput. Chem. 24 (2003) $422-436$.

[28] C. Gatti, P. Fantucci, G. Pacchioni, Charge-density topological study of bonding in lithium clusters.1. planar li ${ }_{n}$ clusters $(\mathrm{n}=4,5,6)$, Theor. Chim. Acta (Berlin) 72 (1987) 433-458.

[29] W. L. Cao, C. Gatti, P. J. MacDougall, R. F. W. Bader, On the presence of nonnuclear attractors in the charge distribution of li and na cluster, Chem. Phys. Lett. 141 (1987) 380.

[30] A. Martín Pendás, M. A. Blanco, A. Costales, P. Mori Sánchez, V. Luaña, Non-nuclear maxima of the electron density, Phys. Rev. Lett. 83 (1999) 1930-1933.

[31] Y. Ma, M. Eremets, A. R. Oganov, Y. Xie, I. Trojan, S. Medvedev, A. O. Lyakhov, M. Valle, V. Prakapenka, Transparent dense sodium, Nature 458 (2009) 182-185

[32] G. K. H. Madsen, C. Gatti, B. B. Iversen, L. Damjanovic, G. D. Stucky, V. I. Srdanov, F center in sodium electrosodalite as a physical manifestation of a non-nuclear attractor in the electron density, Phys. Rev. B 59 (1999) 12359-12369.

[33] P. Mori-Sánchez, J. M. Recio, B. Silvi, C. Sousa, A. Martín Pendás, V. Luaña, F. Illas, The rigorous characterization of mgo $f$ centers as pseudoatoms., Phys. Rev. B 66 (2002) 075103.

[34] Q. K. Timerghazin, G. H. Peslherbe, Electronic structure of the acetonitrile and acetonitrile dimer anions: A topological investigation, The Journal of Physical Chemistry B 112 (2) (2008) 520-528.

[35] M. García-Revilla, E. Francisco, P. L. A. Popelier, A. Martín Pendás, Domain-averaged exchange-correlation energies as a physical underpinning for chemical graphs, ChemPhysChem 14 (6) (2013) 1211-1218.

[36] A. Martín Pendás, M. A. Blanco, E. Francisco, The nature of the hydrogen bond: A synthesis from the interacting quantum atoms picture, The Journal of Chemical Physics 125 (18) (2006) 184112.

[37] J. Meister, W. H. E. Schwarz, Principal components of ionicity, The Journal of Physical Chemistry 98 (33) (1994) $8245-8252$.

[38] T. Bürgi, J. Dunitz, Structure Correlation, VCH, Weinheim, 1994.

[39] C. Reichardt, Solvents and Solvent Effects in Organic Chemistry, VCH, Weinheim, 1988.

[40] I. Fleming, Frontier orbitals and organic chemical reactions, John Wiley and sons, New York, 1976.

[41] J. Neuss, Aromatizitsät: Geschichte und mathematische Analyse eines fundamentalen chemischen Begriffs, Hyle publications, Karlsruhe, 2002.

[42] R. F. W. Bader, On the non-existence of parallel universes in chemistry, Found. Chem. 13 (2011) 11-37.

[43] R. F. W. Bader, Worlds apart in chemistry: A personal tribute to j. c. slater, J. Phys. Chem. A 115 (2011) 12667-12676.

[44] J.-B. Liu, W. H. E. Schwarz, J. Li, On two different objectives of the concepts of ionic radii, Chemistry - A European Journal 19 (44) (2013) $14758-14767$.

[45] A. Lüchow, R. Petz, Single electron densities: A new tool to analyze molecular wavefunctions, J. Comput. Chem. 32 (2011) $2619-2626$.

[46] J. E. Lennard-Jones, New ideas in chemistry, Advancement of Science 11 (1954) 136-148.

[47] P. Macchi, A. Sironi, Chemical bonding in transition metal carbonyl clusters: complementary analysis of theoretical and experimental electron densities., Coord. Chem. Rev. 238-239 (2003) 383-412.

[48] C. Foroutan-Nejad, S. Shahbazian, R. Marek, Toward a consistent interpretation of the QTAIM: Tortuous link between chemical bonds, interactions, and bond/line paths, Chemistry - A European Journal 20 (2014) 10140-10152.

[49] E. Ramos-Cordoba, E. Matito, I. Mayer, P. Salvador, Toward a unique definition of the local spin, Journal of Chemical Theory and Computation 8 (4) (2012) 1270-1279.

[50] L. J. Farrugia, C. Evans, M. Tegel, Chemical bonds without "chemical bonding"? a combined experimental and theoretical charge density study on an iron trimethylenemethane complex, The Journal of Physical Chemistry A 110 (25) (2006) 7952-7961.

[51] J. Poater, M. Solà, F. M. Bickelhaupt, Hydrogen-hydrogen bonding in planar biphenyl, predicted by atoms-in-molecules theory, does not exist, Chemistry - A European Journal 12 (10) (2006) 2889-2895.

[52] J. Poater, M. Solà, F. M. Bickelhaupt, A model of the chemical bond must be rooted in quantum mechanics, provide insight, and possess predictive power, Chemistry - A European Journal 12 (10) (2006) 2902-2905.

[53] T. Strenalyuk, A. Haaland, Chemical bonding in the inclusion complex of he in adamantane (He $@$ adam): The origin of the barrier to dissociation, Chemistry - A European Journal 14 (33) (2008) 10223-10226.

[54] S. Grimme, C. Mück-Lichtenfeld, G. Erker, G. Kehr, H. Wang, H. Beckers, H. Willner, When do interacting atoms form a chemical bond? spectroscopic measurements and theoretical analyses of dideuteriophenanthrene, Angewandte Chemie International Edition 48 (14) (2009) 
2592-2595.

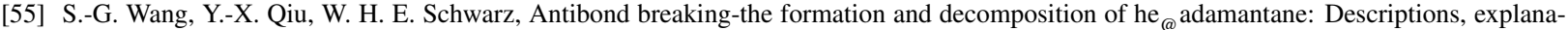
tions, and meaning of concepts, Chemistry - A European Journal 16 (30) (2010) 9107-9116.

[56] J. R. Lane, J. Contreras-García, J.-P. Piquemal, B. J. Miller, H. G. Kjaergaard, Are bond critical points really critical for hydrogen bonding?, Journal of Chemical Theory and Computation 9 (8) (2013) 3263-3266.

[57] G. A. Jeffrey, J. F. Piniella (Eds.), The Application of Charge Density Research to Chemistry and Drug Design, NATO ASI Series, Vol. 250, Plenum Press, New York, 1991.

[58] P. Coppens, X-Ray Charge Densities and Chemical Bonding, International Union of Crystallography Texts on Crystallography, Oxford University Press, Oxford, 1997.

[59] C. F. Matta, R. J. Boyd (Eds.), Wiley-VCH, Weinheim, 2007.

[60] C. Gatti, P. Macchi (Eds.), Modern Charge Density Analysis, Springer-Verlag, Berlin, 2012

[61] D. Stalke (Ed.), Electron Density and Chemical Bonding I, Experimental Charge Density Studies, Structure and Bonding, Vol. 146, SpringerVerlag, Berlin, 2012.

[62] T. S. Koritsanszky, P. Coppens, Chemical applications of x-ray charge-density analysis, Chem. Rev. 101 (2001) $1583-1628$.

[63] W. Scherer, G. S. McGrady, Agostic interactions in $d^{0}$ metal alkyl complexes, Angew. Chem. Int. Ed. 43 (2004) $1782-1806$.

[64] C. Gatti, Chemical bonding in crystals: new directions, Zeit. Kristallogr. 220 (2005) 399-457.

[65] P. Luger, Fast electron density methods in the life sciences-a routine application in the future?, Org. Biomol. Chem. 5 (2007) 2529-2540.

[66] D. Stalke, Meaningful structural descriptors from charge density, Chem. Eur. J. 17 (2011) 9264-9278.

[67] S. Grabowsky, D. Jayatilaka, R. F. Fink, T. Schirmeister, B. Engels, Can experimental electron-density studies be used as a tool to predict biologically relevant properties of low-molecular weight enzyme ligands?, Z. Anorg. Allg. Chem. 639 (2013) 1905-1921.

[68] M. S. Schmøkel, J. Overgaard, B. B. Iversen, Experimental electron density studies of inorganic materials, Z. Anorg. Allg. Chem. 639 (2013) 19221932.

[69] A. Savin, R. Nesper, S. Wengert, T. F. Fässler, ELF: The electron localization function, Angew. Chem. Int. Ed. Engl. 36 (1997) $1809-1832$.

[70] A. R. Oganov1, J. Chen, C. Gatti, Y. Ma, Y. Ma, C. W. Glass, Z. Liu, T. Yu, O. O. Kurakevych, V. L. Solozhenko, Ionic high-pressure form of elemental boron, Nature 457 (2009) 863-868.

[71] T. L. Fletcher, S. M. Kandathil, P. L. A. Popelier, The prediction of atomic kinetic energies from coordinates of surrounding atoms using kriging machine learning, Theor. Chem. Acc. 133 (2014) 1499:1-10.

[72] A. Martín Pendás, E. Francisco, M. A. Blanco, C. Gatti, Bond paths as privileged exchange channels, Chemistry - A European Journal 13 (33) (2007) 9362-9371.

[73] V. Tognetti, L. Joubert, On the physical role of exchange in the formation of an intramolecular bond path between two electronegative atoms, The Journal of Chemical Physics 138 (2) (2013) 024102.

[74] K. Ruedenberg, W. Schwarz, Three millennia of atoms and molecules, in: E. Storm, A. Wilson (Eds.), Pioneers in Quantum Chemistry, ACS Symposium Series, Vol. 1122, ACS, Washington DC, 2013, pp. 1-45.

[75] W. H. E. Schwarz, H. Schmidbaur, Observations and descriptions versus explanations-an example: Does nature, does theory know about steric hindrance?, Chemistry - A European Journal 18 (15) (2012) 4470-4479.

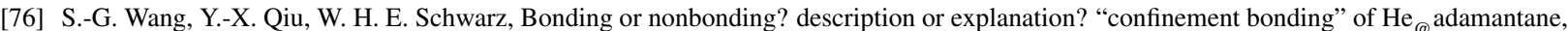
Chemistry - A European Journal 15 (24) (2009) 6032-6040.

[77] P. Lazzeretti, Assessment of aromaticity via molecular response properties, Phys. Chem. Chem.Phys. 6 (2004) 217-223.

[78] S. Shahbazian, M. Zahedi, The role of observables and non-observables in chemistry: A critique of chemical language, Foundations of Chemistry 8 (1) (2006) 37-52.

[79] E. Matito, J. Poater, M. Solà, M. Duran, P. Salvador, Comparison of the AIM delocalization index and the mayer and fuzzy atom bond orders, The Journal of Physical Chemistry A 109 (43) (2005) 9904-9910.

[80] G. Frenking, A. Krapp, Unicorns in the world of chemical bonding models, Journal of Computational Chemistry 28 (1) (2007) 15-24.

[81] S. Grabowsky, R. Kalinowski, M. Weber, D. Förster, C. Paulmann, P. Luger, Transferability and reproducibility in electron-density studies - bond-topological and atomic properties of tripeptides of the type L-alanyl- $X$-L-alanine, Acta Crystallographica Section B 65 (4) (2009) 488-501.

[82] B. Dittrich, C. B. Hübschle, K. Pröpper, F. Dietrich, T. Stolper, J. J. Holstein, The generalized invariom database (GID), Acta Crystallographica Section B 69 (2) (2013) 91-104.

[83] S. Shahbazian, Beyond the orthodox QTAIM: motivations, current status, prospects and challenges, Foundations of Chemistry 15 (2013) 287-302.

[84] M. Goli, S. Shahbazian, Deciphering the "chemical" nature of the exotic isotopes of hydrogen by the MC-QTAIM analysis: the positively charged muon and the muonic helium as new members of the periodic table, Phys. Chem. Chem. Phys. 16 (2014) $6602-6613$.

[85] T. Burnus, M. A. L. Marques, E. K. U. Gross, Time-dependent electron localization function, Phys. Rev. A 71 (2005) 010501.

[86] P.-G. Reinhard, J. A. Maruhn, A. S. Umar, V. E. Oberacker, Localization in light nuclei, Phys. Rev. C 83 (2011) 034312.

[87] V. Polo, J. Andres, S. Berski, L. R. Domingo, B. Silvi, Understanding reaction mechanisms in organic chemistry from catastrophe theory applied to the electron localization function topology, The Journal of Physical Chemistry A 112 (31) (2008) 7128-7136.

[88] V. Polo, P. Gonzalez-Navarrete, B. Silvi, J. Andrés, An electron localization function and catastrophe theory analysis on the molecular mechanism of gas-phase identity $s_{n} 2$ reactions, Theor. Chem. Acc. 120 (2008) 341-349.

[89] M. Deutsch, N. Claiser, S. Pillet, Y. Chumakov, P. Becker, J.-M. Gillet, B. Gillon, C. Lecomte, M. Souhassou, Experimental determination of spin-dependent electron density by joint refinement of X-ray and polarized neutron diffraction data, Acta Crystallographica Section A 68 (6) (2012) 675-686.

[90] K. Boguslawski, C. R. Jacob, M. Reiher, Can dft accurately predict spin densities? analysis of discrepancies in iron nitrosyl complexes, Journal of Chemical Theory and Computation 7 (9) (2011) 2740-2752.

[91] P. G. Mezey, Catchment region partitioning of energy hypersurfaces, i, Theor. Chim. Acta (Berlin) 58 (1981) 309-330

[92] R. J. Gillespie, Covalent and ionic molecules: Why are $\mathrm{BeF}_{2}$ and $\mathrm{AlF}_{3}$ high melting point solids whereas $\mathrm{BF}_{3}$ and $\mathrm{SiF}_{4}$ are gases?, $\mathrm{Journal}$ 
of Chemical Education 75 (7) (1998) 923.

[93] P. Bultinck, R. Vanholme, P. L. A. Popelier, F. De Proft, P. Geerlings, High-speed calculation of AIM charges through the electronegativity equalization method, The Journal of Physical Chemistry A 108 (46) (2004) 10359-10366.

[94] A. Martín Pendás, M. A. Blanco, E. Francisco, Steric repulsions, rotation barriers, and stereoelectronic effects: A real space perspective, Journal of Computational Chemistry 30 (1) (2009) 98-109.

[95] E. Francisco, A. Martín Pendás, M. A. Blanco, A molecular energy decomposition scheme for atoms in molecules, J. Chem. Theory Comput. 2 (2006) 90-102. 